EPJ Web of Conferences 59, 02014 (2013)

DOI: $10.1051 /$ epjconf/20135902014

(C) Owned by the authors, published by EDP Sciences, 2013

\title{
Preparing for polar-drive ignition on the National Ignition Facility
}

\author{
P.W. McKenty ${ }^{1, a}$, T.J.B. Collins ${ }^{1}$, J.A. Marozas ${ }^{1}$, T.J. Kessler ${ }^{1}$, J.D. Zuegel ${ }^{1}$, \\ M.J. Shoup ${ }^{1}$, R.S. Craxton ${ }^{1}$, F.J. Marshall ${ }^{1}$, A. Shvydky ${ }^{1}$, S. Skupsky ${ }^{1}$, \\ V.N. Goncharov ${ }^{1}$, P.B. Radha ${ }^{1}$, R. Epstein ${ }^{1}$, T.C. Sangster ${ }^{1}$, \\ D.D. Meyerhofer ${ }^{1,2}$, R.L. McCrory ${ }^{1,2}$, J.D. Kilkenny ${ }^{3}$, A. Nikroo ${ }^{3}$, M.L. Hoppe ${ }^{3}$, \\ A.J. MacKinnon ${ }^{4}$, S. LePape ${ }^{4}$, M.M. Marinak ${ }^{4}$, M.J. Schmitt ${ }^{5}$, P.A. Bradley ${ }^{5}$, \\ N.S. Krasheninnikova ${ }^{5}$, G.R. Magelssen ${ }^{5}$ and T.J. Murphy ${ }^{5}$ \\ ${ }^{1}$ Laboratory for Laser Energetics, University of Rochester, Rochester, NY, USA \\ ${ }^{2}$ Also Departments of Mechanical Engineering and Physics \& Astronomy, University of \\ Rochester, Rochester, NY, USA \\ ${ }^{3}$ General Atomics, San Diego, CA, USA \\ ${ }^{4}$ Lawrence Livermore National Laboratory, Livermore, CA, USA \\ ${ }^{5}$ Los Alamos National Laboratory, Los Alamos, NM, USA
}

\begin{abstract}
The implementation of polar drive (PD) at the National Ignition Facility (NIF) will enable the execution of direct-drive implosions while the facility is configured for x-ray drive. The Laboratory for Laser Energetics (LLE), in collaboration with LLNL, LANL and GA, is implementing PD on the NIF. LLE has designed and participates in the use of PD implosions for diagnostic commissioning on the NIF. LLE has an active experimental campaign to develop PD in both warm and cryogenic target experiments on OMEGA. LLE and its partners are developing a Polar Drive Project Execution Plan, which will provide a detailed outline of the requirements, resources, and timetable leading to PD-ignition experiments on the NIF.
\end{abstract}

\section{INTRODUCTION}

Four parallel and independent tasks, required to prepare the National Ignition Facility (NIF) for polardrive (PD)-ignition experiments in the near future, are documented in this article. These tasks include (1) optimization of a PD point design [1]; (2) the implementation of one-dimensional, multiple-frequency modulation smoothing by spectral dispersion (Multi-FM SSD) beam smoothing [2]; (3) the design of new optical elements including continuous phase plates (CPP's) and distributed polarization rotators (DPR's); and (4) the deployment of a new cryogenic target handling and insertion subsystem dedicated to the delivery of a cryogenic PD ignition target to the center of the NIF chamber.

\section{TARGET DESIGN}

LLE has developed a symmetric hot-spot ignition point design that has evolved from an initial "allDT" design [3-5], which was extensively explored numerically by McKenty et al. [6]. Significant

\footnotetext{
e-mail: pmck@lle.rochester.edu
}

This is an Open Access article distributed under the terms of the Creative Commons Attribution License 2.0, which permits unrestricted use, distribution, and reproduction in any medium, provided the original work is properly cited. 


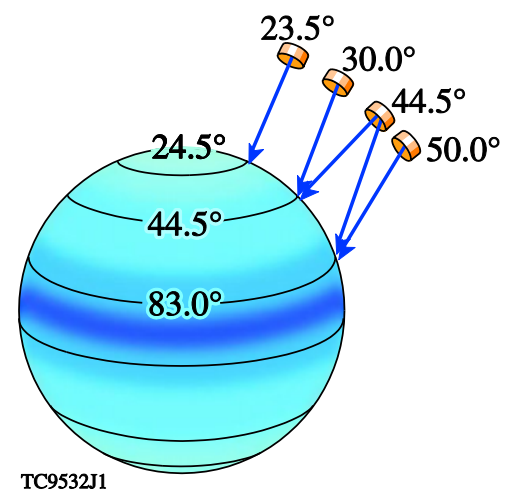

Figure 1. PD pointing configuration for the four rings of NIF beams.

modifications to the design were applied by Goncharov addressing adiabat control [7], shock timing [8], and hot-electron preheat [9]. The point design comprises a cryogenic deuterium-tritium (DT) fuel layer held at just below the triple-point temperature surrounded by a thick $\mathrm{CH}$ ablator. Two-dimensional (2-D) numerical simulations predict a target gain of 45 when all sources of nonuniformities are considered [5].

The PD hot-spot ignition design is modeled after the symmetric design above and achieves a gain of 32 when all sources of nonuniformities are considered [10]. The high gain is maintained in the PD geometry by using appropriately designed phase plates and judicious repointing of the NIF beams. The 192 NIF beams are grouped into four rings of beam ports in each hemisphere having angular offsets of $23.5^{\circ}, 30^{\circ}, 44.5^{\circ}$, and $50^{\circ}$ from the pole. For polar drive, the $30^{\circ}, 50^{\circ}$, and some of the $44.5^{\circ}$ beams are repointed to five positions on target as shown in Fig. 1. Each ring of beams has a unique pulse shape and CPP.

\section{BEAM SMOOTHING}

Polar-drive ignition requires Multi-FM SSD single-beam smoothing, in combination with CPP's and DPR's, to achieve the required irradiation uniformity. MultiFM SSD requires conditioning the laser pulse with three high-frequency modulators to increase the bandwidth followed by a dispersion grating to increase the temporal skew. The large bandwidth and divergence required for Multi-FM SSD would cause the NIF Laser System's limit to be exceeded if it were applied during the main drive pulse. However, as shown in Fig. 2, the full benefit of applying the SSD bandwidth can be achieved during the low-energy picket region of the pulse. As such, the Multi-FM SSD bandwidth need only be applied early in the pulse (over the duration of the pickets) and will not be present during the high-power drive portion of the pulse, thereby alleviating laser-modulation damage issues during the high-energy portion of the pulse.

\section{USER OPTICS}

Beam-smoothing optics are critical to the success of polar-drive ignition. The top and bottom sets of 96 beams are subdivided into five sets of focal spots. Three phase-plate designs produce circularly symmetric focal spots, while the other two phase plates produce skewed, elliptical focal spots (see Fig. 3). Rings 3c and 4c are composed of a superposition of circular and elliptical spots modulated with a spatial mask to ensure correct roll-off at the beam edges. Such shapes provide $\sim 15 \%-20 \%$ improvement in absorption near the target equator.

Additional smoothing of the focal spots, provided by DPR's, is critical for polar-drive target performance. The baseline design includes two $\mathrm{KD} * \mathrm{P}$ half-wave plates, configured in a $2 \times 2$ 


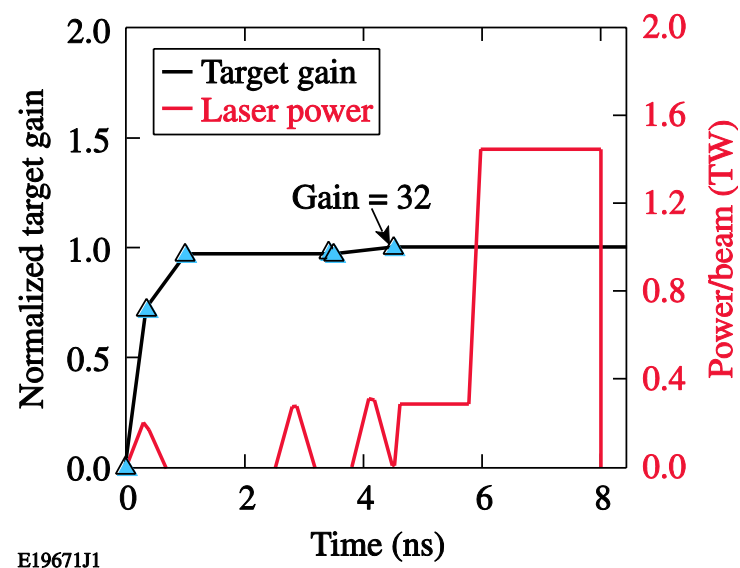

Figure 2. Evaluation of target gain as a function of the application time of Multi-FM SSD bandwidth. Twodimensional DRACO simulations $(\Delta)$ indicate that full target performance is achieved with bandwidth applied until only shortly after the second picket of the laser pulse.

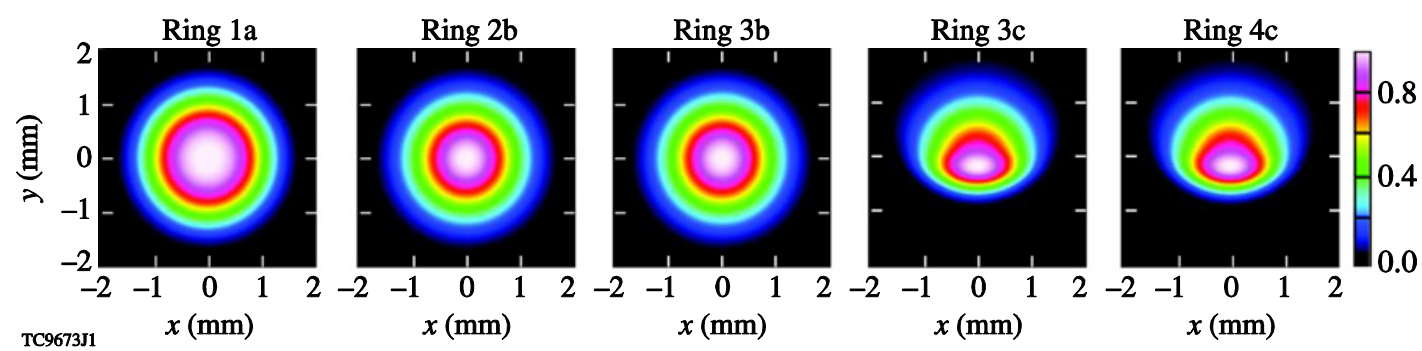

Figure 3. The beam-spot shapes for each ring of the NIF beams.

checkerboard array and placed in each of the 192 final optics assemblies. Since this concept can lead to a loss of energy on target, a parallel technique that employs subwavelength gratings designed to perform as half-wave plates, will be developed to reduce the overall technical risk.

\section{INTEGRATED TARGET INSERTION CRYOSTAT (ITIC)}

The PD-ITIC has been designed to maintain the existing NIF space envelope, while employing several existing NIF systems including the Target Alignment System (TAS) illustrated in Fig. 4; the Load, Layer, and Characterization Station (LLCS); and the cryogenic Target Positioner (cryo-TARPOS) interface.

Other key requirements, including minimizing target exposure to background radiation and the development of a single-sided shroud retractor, will have a substantial impact on the PD-ITIC. These requirements impose severe limitations on the handling of the cryogenic target and the sequencing of pre-shot operations (sub-cooling, alignment, and layering). LLE is deploying the infrastructure needed to ensure options for target survival once exposed to the ambient radiation of the target chamber. Furthermore, LLE will (1) develop the techniques required to layer and characterize an ignition target that has been filled with fuel through a fill tube and (2) develop and test schemes for single-sided shroud retraction. 


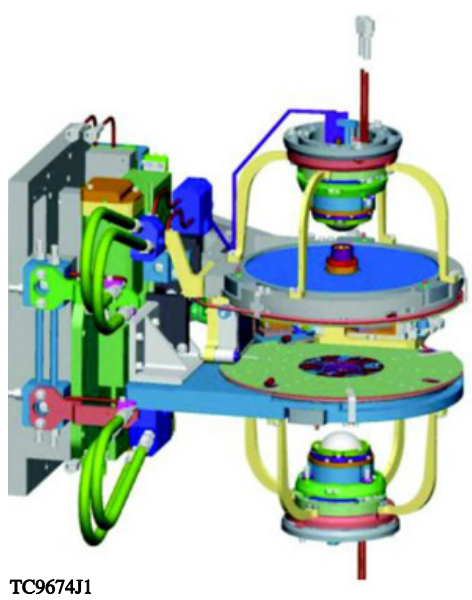

Figure 4. The Target Alignment System used to simultaneously view the target and the location of all 192 beam spots. Note that the tight space constraint limits the overall diameter of the PD ITIC shroud.

This work was supported by the U.S. Department of Energy Office of Inertial Confinement Fusion under Cooperative Agreement No. DE-FC52-08NA28302, the University of Rochester, and the New York State Energy Research and Development Authority. The support of DOE does not constitute an endorsement by DOE of the views expressed in this article.

\section{References}

[1] “Triple Picket Point Design for Polar-Drive Ignition on NIF," S-AA-G-082 Rev. B, Laboratory for Laser Energetics, University of Rochester, Rochester, NY (12 September 2011)

[2] "Multi-FM 1D-SSD Requirements on OMEGA," T-BW-R-005-A, Laboratory for Laser Energetics, University of Rochester, Rochester, NY (14 April 2011)

[3] See National Technical Information Service Order No. DE2002762802 [Laboratory for Laser Energetics LLE Review Quarterly Report 79, 121 (1999)]. Copies may be obtained from the National Technical Information Service, Springfield, VA 22161

[4] S.E. Bodner et al., Phys. Plasmas 5, 1901 (1998)

[5] C.P. Verdon, Bull. Am. Phys. Soc. 38, 2010 (1993)

[6] P.W. McKenty et al., Phys. Plasmas 8, 2315 (2001)

[7] V.N. Goncharov et al., Phys. Plasmas 10, 1906 (2003)

[8] T.R. Boehly et al., Phys. Rev. Lett. 106, 195005 (2011)

[9] V.N. Goncharov et al., Phys. Plasmas 15, 056310 (2008)

[10] T.J.B. Collins et al., "Preparing for Polar Drive at the National Ignition Facility," to be published in Physics of Plasmas. 